\title{
Adoption of focused ultrasound thalamotomy for essential tremor: why so much fuss about FUS?
}

\author{
Christian lorio-Morin 다, ${ }^{1}$ Mojgan Hodaie $\quad$ (), ${ }^{2}$ Andres M Lozano ${ }^{2}$
}

${ }^{1}$ Neurosurgery, Faculté de médecine et des sciences de la santé, Université de Sherbrooke, Sherbrooke, Québec, Canada ${ }^{2}$ Division of Neurosurgery, Toronto Western Hospital, Toronto, Ontario, Canada

\section{Correspondence to} Dr Christian lorio-Morin, Neurosurgery, Universite de Sherbrooke Faculte de medecine et des sciences de la sante, Sherbrooke, Quebec, Canada; christian.iorio-morin@ usherbrooke.ca

Received 30 May 2020 Revised 27 November 2020 Accepted 22 December 2020 Published Online First 9 February 2021

\section{Linked}

- http://dx.doi.org/10.1136/ jnnp-2021-326031

Check for updates

(C) Author(s) (or their employer(s)) 2021. No commercial re-use. See rights and permissions. Published by BMJ.

To cite: lorio-Morin C, Hodaie M, Lozano AM. J Neurol Neurosurg Psychiatry 2021:92:549-554.
ABSTRACT
Background Focused ultrasound (FUS) was approved as a new treatment modality for essential tremor (ET) in 2016. The goal of this study was to quantify FUS adoption for ET and understand its drivers.

Methods The adoption of the various surgical options for ET was estimated using three measures: the number of presentations on the various surgical treatments for ET at specialised international meetings, the number of original papers published as identified by literature searches and the number of thalamotomy procedures performed worldwide for ET as provided by device manufacturers' registries.

Results First, we found that the number of presentations related to lesioning procedures is increasing relative to deep brain stimulation (DBS) at international meetings. Second, there are already more publications on FUS (93) than stereotactic radiosurgery (SRS) (68) or radiofrequency (43) for ET, although they still lag behind DBS papers (750). Third, the number of annual FUS thalamotomies performed for ET ( $n>1200$ in 2019) in 44 centres has surpassed the annual procedures across 342 Gamma Knife units $(n<400,2018)$ but is yet to reach the number of DBS cases for ET estimated at over 2400/year.

Conclusion FUS is being rapidly adopted for the treatment of ET. We hypothesise that its perceived minimally invasive nature coupled with the ability to perform intraoperative clinical assessments, its immediate effects and active marketing efforts are contributing factors. As lesioning modalities for the treatment of ET are reappraised, the superior popularity of FUS over SRS appears to arise for reasons other than differences in clinical outcomes.

\section{INTRODUCTION}

The surgical treatment of medically refractory essential tremor (ET) currently revolves around two approaches: neuromodulation through deep brain stimulation (DBS) and stereotactic lesioning of the ventral intermediate (VIM) nucleus of the thalamus (thalamotomy). ${ }^{1}$ Thalamotomies can be performed using various techniques including invasive radiofrequency thermocoagulation (RF) and laser interstitial thermal therapy (LITT), or non-invasive Gamma Knife (GK) stereotactic radiosurgery (SRS) and magnetic resonance-guided focused ultrasound (FUS). The latter is the newest method and was approved by the US Food and Drug Administration (FDA) in 2016 for the unilateral treatment of tremor. ${ }^{2}$ When considering surgical treatment for ET, movement disorder neurologists refer their patients to neurosurgeons who might use either a single or several of these techniques. Patients might also have a specific preference based on scientific, marketing material or social networking they may have been exposed to.

Adoption of new technologies typically occurs in a pattern, from innovators and early adopters, to the early majority, majority and non-adopters. ${ }^{3}$ The speed and breadth of adoption is influenced by many factors, including the effectiveness of the new technology, the advantages it provides over competing products, its cost, availability, its ease of use and the aggressiveness with which the product is marketed. Surgery for movement disorders is among the most technology-vested fields of medicine and has seen important innovation (eg, neuronavigation, DBS). Understanding the reasons why a specific technology is being adopted can help identify breakthroughs in the constant stream of new product introductions. Conversely, this understanding should help protect patients and the health system from unsubstantiated treatments and trends.

We are under the impression that the development of FUS renewed the overall interest of the functional neurosurgery community in lesioning procedures relative to DBS, and that its adoption is faster and broader than that observed for similar modalities, most specifically GK. The goal of this study was to (1) quantify the current interest in lesioning procedure for ET, (2) compare the adoption of FUS thalamotomies for tremor to other treatment modalities and (3) examine the possible reasons underlying the trajectory of the adoption of FUS.

\section{METHODS}

\section{Measuring the interest in FUS}

Neurosurgeon's overall interest in lesioning procedures relative to neuromodulation was first assessed by analysing the published proceedings from the American Society for Stereotactic and Functional Neurosurgery (ASSFN) and the European Society for Stereotactic and Functional Neurosurgery (ESSFN) meetings between 2010 and 2019.45 The abstract of each oral presentation was read to identify the use of lesioning (RF, SRS, LITT, FUS, other or lesioning using an unspecified modality) or neuromodulation techniques (DBS or other) for any indication. Abstracts reporting multiple modalities were assigned to more than one category.

\section{Using publications as a proxy for interest and adoption}

Specific interest in the technique used for thalamotomy in ET was assessed by counting the number 
Table 1 Publications on essential tremor stratified by treatment modality

\begin{tabular}{|c|c|c|c|c|}
\hline & Deep brain stimulation* & Focused ultrasoundt & $\begin{array}{l}\text { Stereotactic } \\
\text { radiosurgery } ¥\end{array}$ & $\begin{array}{l}\text { Radiofrequency } \\
\text { thermocoagulation§ }\end{array}$ \\
\hline Number of original articles (excluding reviews) & 750 & 93 & 68 & 43 \\
\hline Publication years & 1993-2019 & 2010-2019 & 1992-2019 & 1991-2019 \\
\hline Level of evidence (Oxford Centre for Evidence-Based Medicine) ${ }^{9}$ & Level 2 & Level 2 & Level 3 & Level 3 \\
\hline
\end{tabular}

of annual publications for each modality. The Scopus database was searched using 'Essential tremor' and keywords for each modality ("radiofrequency" OR "thermocoagulation", "deep brain stimulation", "radiosurgery" OR "Gamma Knife", "focused ultrasound"). Complete queries are listed in the footnotes of table 1 . The search was performed on 20 April 2020 and restricted to the title, abstract and keywords of papers published before 2020. We included only original articles and excluded reviews.

\section{Measures of adoption}

Adoption of the various lesioning techniques was assessed by contacting vendors of each modality and requesting the number of active clinical units, as well as patients treated for ET using their technology. FUS data were provided by Insightec (Tirat Carmel, Israel). GK data were provided by the Leksell Gamma Knife Society (Stockholm, Sweden). The number of DBS procedures performed for ET was not available. The rate of DBS implantation for ET was therefore estimated based on the 2011 US rate of 0.45 per 100000 population, ${ }^{6}$ a 2019 US population of 327.5 million $^{7}$ and an assumption that the US might represent $40 \%$ of the total implantation rate. ${ }^{8}$ The lower margin assumes that the implantation rate for ET did not increase between 2011 and 2019 and that FUS thalamotomy patients would have otherwise had a DBS implantation. The upper margin assumes that the DBS implantation rate increased between 2011 and 2019 and that the FUS thalamotomies are performed in patients who are not eligible for DBS. It should be noted that this estimate remains approximative at best.

\section{Measures of safety and efficacy}

To assess the safety and efficacy of the various procedures, the search strategy detailed above was performed again for reviews. Abstracts were screened and the latest appropriate reviews selected to inform the discussion and generate hypotheses on FUS adoption.

\section{RESULTS \\ Interest in lesioning procedures at functional neurosurgery meetings}

Between 2010 and 2020, 10 meetings of the ASSFN or ESSFN were held (figure 1). An average of 52 and 178 oral presentations were given at each ASSFN or ESSFN meeting, respectively. While lesioning (all techniques and indications combined) represented a minority of the discussions, the proportion of oral presentations attributed to lesioning increased from $2 \%$ $(1 / 64)$ at the 2010 ASSFN meeting to $25 \%(11 / 44)$ at the 2018 edition (figure 1A). This trend was not observed in the ESSFN meetings, which consistently had 11\%-14\% (20-27/91-105) of oral presentations related to lesions (figure $1 \mathrm{~B}$ ). The increased number of presentations on lesions at American meetings spanned across all modalities with presentations on RF $(n=1)$, SRS $(n=6)$, LITT $(n=2)$ and FUS $(n=2)$ in 2018 (figure 1C). The indications for these functional procedures varied by meeting and modality, but included epilepsy, psychiatric disease and movement disorders, of which ET was the most represented condition.
A

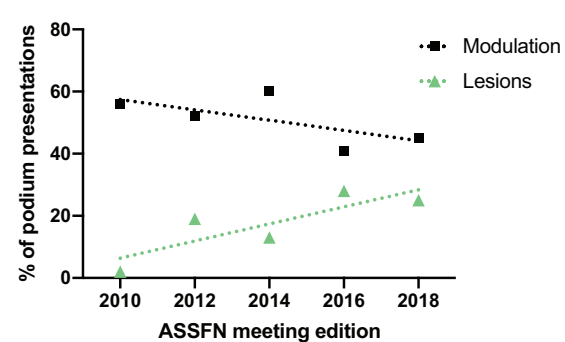

B

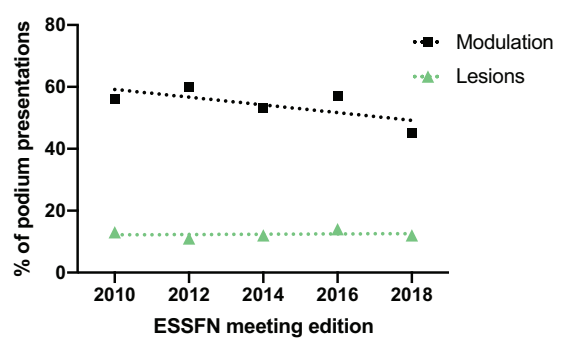

C

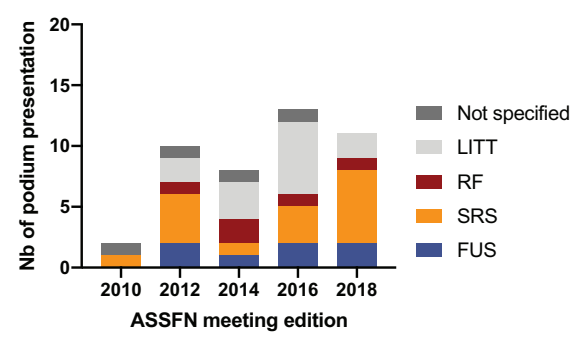

Figure 1 Oral presentations at functional neurosurgery meetings. Percentage of podium presentations attributed to modulation (eg, deep brain stimulation or other) or lesioning techniques (eg, RF, SRS, FUS, LITT, other or not specified) with linear regression (dashed lines) between the 2010 and 2019 editions of international meetings. The data were collected from each meeting's proceedings. (A) At the ASSFN meeting, the proportion of oral presentations attributed to lesioning increased from 2\% (1/64) in 2010 to 25\% (11/44) in 2018. (B) At the ESSFN meeting, 11\%-14\% (20-27/91-105) of oral presentations were attributed to lesioning between 2010 and 2018. There was no evidence of an increasing trend. (C) Detail of lesioning modalities discussed at the ASSFN meetings. The increasing interest was observed across all modalities and not limited to a single technique. ASSFN, American Society for Stereotactic and Functional Neurosurgery; ESSFN, European Society for Stereotactic and Functional Neurosurgery; FUS, focused ultrasound; LITT, laser interstitial thermal therapy; RF, radiofrequency thermocoagulation; SRS, stereotactic radiosurgery. 


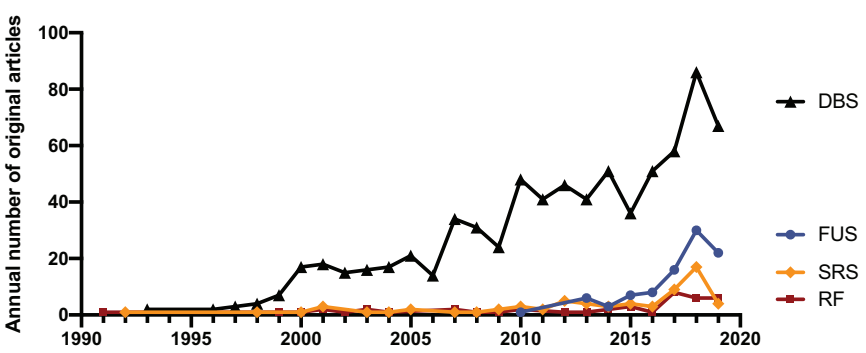

Figure 2 Annual publications on ET stratified by treatment modality. The Scopus database was searched on 20 April 2020 for original articles (excluding reviews) published before 2020 and discussing ET and each modality (see footnote of table 1 for complete queries). DBS had the highest number of publications (750). FUS had 93 publications and a pattern mirroring that of DBS, with 50 publications achieved within 8 years of the modality's first paper. Both SRS (68 publications) and RF (43 publications) experienced an increase in annual publications in 2016-2019 after 20 years of steady state. DBS, deep brain stimulation; ET, essential tremor; FUS, focused ultrasound; RF, radiofrequency thermocoagulation; SRS, stereotactic radiosurgery.

\section{Modality-stratified publications on ET}

The literature on the surgical treatment of ET shows an increase in interest in lesion surgery, particularly FUS for ET (figure 2). Between 1991 and 2019, 750 original articles (excluding reviews) were published on DBS for ET versus 43 on RF, 68 on SRS and 93 on FUS. In 2010, 11\% of surgical papers on ET were on a lesioning technique, a number that almost tripled to $32 \%$ in 2019. Within the lesioning techniques, the annual number of publications on FUS has been equal to or greater than both SRS and RF every year since 2013. The time required to reach the 50 th publication from the first paper was 8 years for DBS for ET and similarly 8 years for FUS. This suggests that the rate of adoption of FUS for ET may be similar to that which occurred for DBS. In contrast, it took 26 years to accumulate 50 publications on SRS for ET and this level has still not been reached for RF (>28 years). It took 8 years for the FUS literature to reach the current total number of SRS papers on ET to date (published between 1992 and 2019).

The quality of the published studies also varied by modality (table 1). There was no level 1 evidence for efficacy in ET based on the Oxford Centre for Evidence-Based Medicine (OCEBM) definition (systematic review of multiple randomised controlled trials). ${ }^{9}$ Level 2 evidence (single randomised controlled trial) was available for DBS $^{10}$ and FUS, ${ }^{11}$ while level 3 evidence (nonrandomised controlled cohort) was available for SRS ${ }^{12}$ and $\mathrm{RF}^{13}$

\section{Adoption of FUS versus GK for the treatment of ET}

The larger number of publications on FUS relative to other modalities is correlated to a wider use of this technology for lesioning in tremor (figure 3). Data provided by the Leksell Gamma Knife Society (self-reported by participating centres between 2011 and 2018 and reflecting 87\%-95\% of GK units sold) and Insightec (reflecting 100\% of treatments performed on clinical FUS units between 2015 and 2019) reveals that over 1200 FUS thalamotomies were performed for ET in the year 2019 year compared with under 400 for GK in 2018. GK thalamotomies have recently increased in 2018 after 5 years of steady state. This could possibly be related to a halo effect of the 2016 FDA approval of FUS ${ }^{2}$ following publication of the pivotal trial. ${ }^{11}$ While the GK data are not yet available for 2019, we have no indication that it increased to the same extent as FUS between

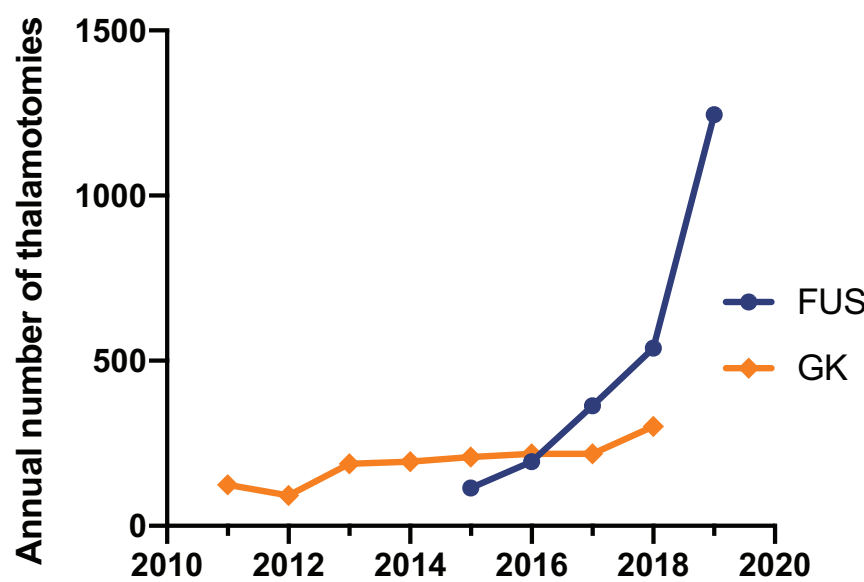

Figure 3 Annual number (worldwide) of thalamotomies performed for ET using FUS or GK. The Leksell Gamma Knife Society and Insightec were contacted to obtain from their registries the number of thalamotomies annually performed worldwide using GK and FUS, respectively. GK data were available between 2011 and 2018 and consisted of self-reported cases from $87 \%$ to $95 \%$ of GK centres. Even when adjusting for centres who did not report their data, the number of GK thalamotomies performed in 2018 was under 400. FUS data were available between 2015 and 2019 and reflected $100 \%$ of treatments performed on clinical FUS units. Over 1200 FUS thalamotomies for ET were reported in the year 2019. ET, essential tremor; FUS, focused ultrasound; GK, Gamma Knife.

2018 and 2019. For comparison, we estimate that annual worldwide DBS implantations for ET might range from 2400 to 4800.

As of March 2020, there were 342 active clinical GK units worldwide (personal communication, Leksell Gamma Knife Society) compared with 44 clinical treatment sites for FUS. ${ }^{14}$ This would imply a worldwide annual rate of 1 thalamotomy per active GK unit per year, compared with 28 per active FUS treatment centre.

\section{DISCUSSION}

From the above data, it is clear that interest in all lesioning techniques for ET has increased in the last 10 years (figure 1), and that FUS thalamotomies are being adopted faster than GK ever was (figure 3). This is occurring despite unclear reimbursement policies in many countries ${ }^{15}$ and a lack of acceptance of bilateral treatments, (as opposed to GK where there are precedents for bilateral interventions. ${ }^{16-18}$ The increased adoption is associated with a higher number of publications (figure 2) as well as higher-quality evidence for FUS treatments (table 1). Despite the fact that SRS has been available longer than DBS and arguably provides non-invasive lesion generation capabilities similar to FUS, the enthusiasm for GK has remained fairly moderate. In the following discussion, we examine the possible contributors to the stalling of SRS techniques and the rapid adoption and growth FUS for ET (table 2).

Hypothesis 1: could the intraoperative clinical assessment possible in FUS be more attractive to functional neurosurgeons than SRS workflow?

Both FUS and SRS allow the generation of lesions without skin incision, burr hole and brain penetration. A significant advantage of FUS over SRS is that it allows graded, incremental lesions that can be titrated to the clinical effects seen during the procedure. FUS lesions are created through successive sonication of increasing power between which the awake patient is typically 
Table 2 Hypotheses explaining FUS adoption

\begin{tabular}{ll}
\hline Can explain FUS adoption over GK & Cannot explain FUS adoption over GK \\
\hline Intraoperative clinical assessment & Effectiveness \\
Device maker's interest in ET & Safety \\
Key opinion leaders' interest in FUS & Patient experience \\
& Cost \\
\hline
\end{tabular}

ET, essential tremor; FUS, focused ultrasound; GK, Gamma Knife.

assessed for efficacy and side effects. This is akin to progressively increasing lesions during conventional RF thalamotomy procedures. With SRS, on the other hand, GK requires acceptance that the lesion and its effect will be delayed, as its effect only appears after a median of 4 months, sometimes up to a year. ${ }^{12} 16$ 19-24 To some patients and functional neurosurgeons, this might be suboptimal, given the highly variable response that can be observed with both modalities (figure 4).

\section{Hypothesis 2: could FUS be more effective than SRS for} tremor?

There is no study directly comparing the outcomes of FUS versus SRS thalamotomies. In the most recent review of surgical outcomes in ET, the rate of tremor control at 12 months was $35 \%-75 \%$ for FUS versus $48 \%-63 \%$ for SRS. ${ }^{1}$ These compare to a $49 \%-100 \%$ historical rate for RF thalamotomies.

The highest-quality study on FUS is an international randomised, sham-controlled trial. ${ }^{11}$ Seventy-six patients with medically refractory ET were assigned to either a unilateral VIM thalamotomy or a sham treatment and followed for 12 months. Crossover was allowed after 3 months for the sham patients. The primary outcome was the between-group difference in the change from baseline to 3 months in hand tremor. Thalamotomy patients showed significantly better tremor reduction (47\% improvement vs $0.1 \%)$, improvement in activities of daily living (62\% improvement vs $3 \%)$ and quality of life $(46 \%$ improvement vs 3\%). These results were sustained at the 3 -year follow-up. ${ }^{25}$ While the goal of this trial was to test FUS lesions specifically, it also provided the highest level of evidence yet for the use of thalamotomies in ET (OCEBM level 2).

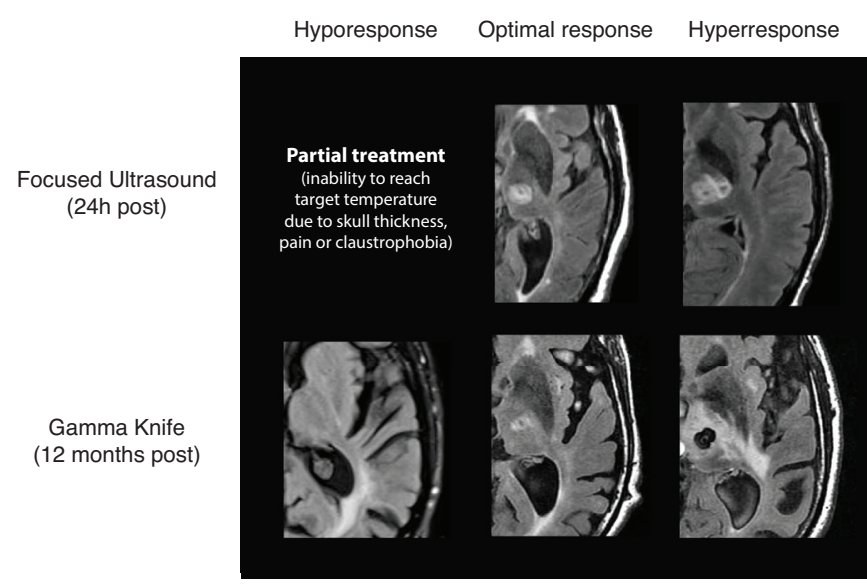

Figure 4 Typical response patterns after left ventral intermediate (VIM) thalamotomy with either focused ultrasound (FUS) or Gamma Knife (GK). Representative fluid-attenuated inversion recovery sequence of responses obtained 24 hours (for FUS) or 12 months (for GK) after left VIM thalamotomy for essential tremor. The protocol used was the same for all treatments of the same modality, highlighting the variability of individual responses.
All SRS thalamotomy data in ET are from single-arm GK series. The highest-quality study is a prospective cohort of 50 patients who underwent unilateral VIM thalamotomy and were followed for 12 months. ${ }^{12}$ Blinded video assessment demonstrated a tremor score improvement of 54\%. Activities of daily living improved by $72 \%$.

While the current evidence convincingly demonstrates that thalamotomies are effective at reducing tremor, the superiority of one modality over the other cannot be established. Because all techniques appear to produce similar improvements, the rapid rate of adoption of FUS is not related to improved outcomes.

\section{Hypothesis 3: could FUS be safer than SRS?}

As for efficacy, there are no studies comparing the safety of FUS versus SRS thalamotomies. In the FUS pivotal trial, adverse events included gait disturbance $(36 \%)$, paresthesia or numbness (38\%) and haemiparesis (4\%) that persisted at 12 months in $9 \%, 14 \%$ and $2 \%$ of patients, respectively. ${ }^{11}$ The median rate of permanent gait disturbance, paresthesia and haemiparesis across all FUS series was 4\%,8\% and 2\%, respectively. ${ }^{1}$ These side effects are similar to those reported for historical RF treatments, which included transient paresthesias (42\%), transient paresis (34\%) and transient dysarthria (29\%). ${ }^{1}$ For SRS series, the median rate of adverse events was 2\% (range 0\%-9\%) for transient symptoms and $0 \%$ (range 0\%-18\%) for permanent symptoms. ${ }^{1}$ In the highest-quality SRS study, no permanent adverse event occurred and a single case of transient haemiparesis (2\%) was documented. ${ }^{12}$ Because most SRS studies are retrospective, adverse events might be underreported. However, even when considering only the prospective studies, the complication rate of FUS treatments appears substantially higher than for GK. The accelerated adoption of FUS over GK cannot be explained on the basis of safety.

Hypothesis 4: could FUS deliver a better patient experience? If safety and efficacy are comparable, neurologists and neurosurgeons could choose the modality that provides the best patient experience. This is a highly subjective, yet important outcome. FUS but not SRS requires complete head shaving. Both FUS and GK require the application of a stereotactic frame. For FUS, the patient is next brought into the MRI suite where he needs to stay in the magnet for usually $2-3$ hour. This can be challenging for patients with claustrophobia, obesity, kyphosis or back pain. During the sonication, a scalp heating sensation can be felt. In a small number, this heating reaches a level of $10 / 10$ or unbearable pain. SRS procedures only last 1 hour, do not require hair shaving, are less claustrophobic and the treatment does not generate any physical sensations. Some patients may be reluctant to receive radiation perhaps due to a perceived associated delayed risk. On the current level of analysis, it would appear that FUS procedures are more involved, take longer and can produce more discomfort than SRS thalamotomies. Their rapid adoption is not due to improved tolerability of the procedure.

\section{Hypothesis 5: is FUS less expensive than GK?}

Adoption of a new treatment could be driven by its lower cost. It is, however, difficult to generalise and estimate the total costs and cost per case of FUS versus SRS because of the large variability in the usage of these modalities across centres. This is largely related to the fact that FUS is restricted to one approved indication while SRS is used primarily for oncology, trigeminal neuralgia and arteriovenous malformations rather than movement disorders. Both procedures require specialised personnel 
and service and are associated with significant capital costs and, in the case of GK, replacement costs of the radioactive sources every 5 years. The costs analysis thus has to be individualised and will likely be driven in large part by the volume of ET cases relative to other indications. The assessment of the cost effectiveness of FUS and SRS for ET will require a separate analysis. Interestingly, both lesioning modalities have been suggested to be more cost-effective than DBS, ${ }^{15}$ and both are probably comparable or more expansive than conventional RF.

\section{Hypothesis 6: could FUS have better marketing than SRS for ET?}

Because tremor is currently the only approved indication for cerebral FUS, manufacturers have higher incentives to aggressively promote and focus on ET. In contrast, ET represents only $0.3 \%$ of annual GK cases $^{26}$ and receives correspondingly little attention from the manufacturer. This is evident in the direct patient outreach performed by the FUS foundation ${ }^{27}$ which has no equivalent in the SRS world. This interest also translates into more funding for clinical trials. As of 20 April 2020, Insightec was documented as a sponsor of 12 of 155 studies on ET registered on clinicaltrial.gov compared with 0 for Elekta, which explains the higher quality of evidence for FUS. Indeed, while performing a modern, randomised, sham-controlled trial of RF thalamotomy might be ethically challenging, there is no obvious reason other than funding and sponsor interest explaining why such a trial was never performed for SRS. Quality studies, in turn, can generate further interest in the field. An additional factor may be related to leadership and direction of therapeutic programmes. In Canada for example, all four FUS centres are led by functional neurosurgeons, compared with only one of the six GK centres where oncological neurosurgeons and radiation oncologists are predominant. The oversight of FUS programmes by functional neurosurgeons and their close interactions with movement disorder neurologists might further drive the adoption of this technology.

\section{What happened to RF?}

The lack of registry for RF thalamotomies (as opposed to Insightec's FUS registry and the Leksell Gamma Knife Society's GK registry) did not allow us to reliably estimate the number of RF thalamotomies performed annually (figure 3 ). Based on the number of publications, however, it is unlikely that RF thalamotomies experienced a resurgence as significant as the rise of FUS (figure 2). It is paradoxical that RF was abandoned two decades ago because DBS was shown to be safer, while FUS is now expanding despite a side effect profile in the $36 \%-38 \%$ range. $^{11}$ It is clear that not all patients desire a permanent implant or are eligible for it. The resurrection of lesioning modalities now provides options to this population who might have been underserved in the 'DBS-only' era. From a cost-effectiveness and accessibility standpoints, RF probably remains advantageous. Whether this modality will keep its relevance in the face of FUS and SRS, which both forgo the need for incision, burr hole and brain penetration remains to be seen.

\section{Limitations}

The discussion of the drivers of the adoption of FUS and SRS for ET is based on the best available data for each modality. Because there is no prospective trial directly comparing SRS and FUS, conclusions regarding efficacy, safety, patient experience and cost might be influenced by the selection bias of each study. For instance, when analysing the studies selected in the latest systematic review of surgical ET management, ${ }^{1}$ the weighted mean (SD) age of patients in the FUS studies ${ }^{11} 25$ 28-33 was 69.2 (3.2) versus 79.4 (3.4) for GK ${ }^{12} 1619-24$ (unpaired t-test $\mathrm{p}=0.0131)$. GK patients were not only older, but also used anticoagulant medication in up to $26 \%$ of cases, ${ }^{19}$ whereas this is currently a contraindication to FUS. Interestingly, one would expect these differences to increase the morbidity of GK relative to FUS. The prospective and controlled nature of the FUS pivotal trial, ${ }^{11}$ which is also the largest FUS study, might have counterbalanced this selection bias by better ascertainment of adverse events compared with GK retrospective series.

\section{CONCLUSION}

The interest in lesioning procedures for functional indications has increased in the last decade. For ET, this interest appears to be driven by the rise of FUS. Three years after its FDA approval, FUS has already surpassed SRS as assessed by the number of research papers and treated patients. This rapid adoption cannot be explained by efficacy, safety, patient experience or cost factors. We hypothesise that the ability to perform intraoperative clinical assessments, the manufacturers' interest in functional neurosurgery as well as functional neurosurgeons' involvement and leadership in the technology are important drivers of FUS uptake. As lesioning modalities are reappraised for the treatment of ET, the relative merits of FUS, SRS and RF should be further considered, as the superior popularity of FUS does not appear to be the result of differences in clinical outcomes. Moving forward, the debate around FUS versus GK versus RF thalamotomies will likely revolve around two key issues: (1) Can GK produce lesions that are predictable and consistent enough in dimension and location to nullify the theoretical benefit of FUS' and RF's intraprocedural clinical assessment? (2) Can one of the three techniques allow safe bilateral treatments? These findings should be considered when counselling patients on a choice of lesioning technique for ET.

Twitter Mojgan Hodaie @mhodaie

Contributors $\mathrm{Cl}-\mathrm{M}$ designed the study, performed the data collection and statistical analysis, and wrote the first draft of the manuscript. MH critically reviewed the manuscript. AML designed the study and critically reviewed the manuscript.

Funding The authors have not declared a specific grant for this research from any funding agency in the public, commercial or not-for-profit sectors.

Competing interests CI-M is founder and CEO of Hyperexis and Abaxial Médical, which are both unrelated to the current study. MH is the Surgical Codirector of the Toronto Western Hospital, Joey \& Toby Tanenbaum Family Gamma Knife Centre. AML is the Director of the Toronto Western Hospital Focused Ultrasound Program, Scientific Director for Functional Neuromodulation and consultant for Medtronic, Abbott, Boston Scientific and Insightec.

Patient consent for publication Not required.

Ethics approval This study did not involve patient data and did not require ethics approval.

Provenance and peer review Not commissioned; externally peer reviewed Data availability statement Data are available upon reasonable request.

\section{ORCID iDs}

Christian lorio-Morin http://orcid.org/0000-0003-2995-7255

Mojgan Hodaie http://orcid.org/0000-0001-6278-4929

\section{REFERENCES}

1 Dallapiazza RF, Lee DJ, De Vloo P, et al. Outcomes from stereotactic surgery for essential tremor. J Neurol Neurosurg Psychiatry 2019;90:474-82.

2 U.S. Food and Drug Administration. FDA approves first MRI-guided focused ultrasound device to treat essential tremor. FDA [Internet], 2016. Available: https://www.fda.gov/ news-events/press-announcements/fda-approves-first-mri-guided-focused-ultrasounddevice-treat-essential-tremor 
3 Beal GM, Bohlen JM. The Diffusion Process [Internet], 1957. Available: http://lib.dr. iastate.edu/specialreports/24

4 American Society for Stereotactic and Functional Neurosurgery. Past Meeting Abstracts [Internet], 2018. Available: http://www.assfn.org/meetings.html?s=2 [Accessed cited 2020 Apr 20].

5 European Society for Stereotactic and Functional Neurosurgery. Past ESSFN Congresses [Internet], 2018. Available: http://www.essfn.org/en/congress-courses/ past/ [Accessed cited 2020 Apr 20].

6 Kalakoti P, Ahmed O, Bollam P, et al. Predictors of unfavorable outcomes following deep brain stimulation for movement disorders and the effect of hospital case volume on outcomes: an analysis of 33,642 patients across 234 US hospitals using the National (nationwide) inpatient sample from 2002 to 2011. Neurosurg Focus 2015:38:E4.

7 United States Census Bureau. U.S. and World Population Clock [Internet]. Available: https://www.census.gov/popclock/ [Accessed cited 2020 May 15].

8 Abosch A, Timmermann L, Bartley S, et al. An international survey of deep brain stimulation procedural steps. Stereotact Funct Neurosurg 2013;91:1-11.

9 OCEBM Levels of Evidence Working Group. The Oxford Levels of Evidence 2 [Internet]. Oxford Centre for Evidence-Based Medicine, 2011. Available: https://www.cebm.net/ index.aspx?o $=5653$

10 Schuurman PR, Bosch DA, Bossuyt PM, et al. A comparison of continuous thalamic stimulation and thalamotomy for suppression of severe tremor. N Engl J Med 2000;342:461-8.

11 Elias WJ, Lipsman N, Ondo WG, et al. A randomized trial of focused ultrasound thalamotomy for essential tremor. N Engl J Med 2016;375:730-9.

12 Witjas T, Carron R, Krack P, et al. A prospective single-blind study of gamma knife thalamotomy for tremor. Neurology 2015;85:1562-8.

13 Zirh A, Reich SG, Dougherty PM, et al. Stereotactic thalamotomy in the treatment of essential tremor of the upper extremity: reassessment including a blinded measure of outcome. J Neurol Neurosurg Psychiatr 1999;66:772-5.

14 Focused Ultrasound Foundation. Treatment Sites - Commercial Treatments and Clinical Trials [Internet]. FUS Foundation, 2020. Available: https://www.fusfoundation.org/ the-technology/treatment-sites?indication=Essential\%20Tremor [Accessed cited 2020 May 4].

15 Ravikumar VK, Parker JJ, Hornbeck TS, et al. Cost-Effectiveness of focused ultrasound, radiosurgery, and DBS for essential tremor. Mov Disord 2017;32:1165-73.

16 Young RF, Li F, Vermeulen S, et al. Gamma knife thalamotomy for treatment of essential tremor: long-term results. J Neurosurg 2010;112:1311-7.

17 Young RF, Hesselgesser RD, Ahn E, et al. Bilateral gamma knife thalamotomy for treatment of axial tremor. Translational Cancer Research 2014;3:525-9.
18 Niranjan A, Raju SS, Monaco EA, et al. Is staged bilateral thalamic radiosurgery an option for otherwise surgically ineligible patients with medically refractory bilateral tremor? J Neurosurg 2018;128:617-26.

19 Kondziolka D, Ong JG, Lee JYK, et al. Gamma knife thalamotomy for essential tremor. J Neurosurg 2008;108:111-7.

20 Lim S-Y, Hodaie M, Fallis M, et al. Gamma knife thalamotomy for disabling tremor: a blinded evaluation. Arch Neurol 2010;67:584-8.

21 Ohye C, Higuchi Y, Shibazaki T, et al. Gamma knife thalamotomy for Parkinson disease and essential tremor: a prospective multicenter study. Neurosurgery 2012;70:526-36. discussion535-6.

22 Kooshkabadi A, Lunsford LD, Tonetti D, et al. Gamma knife thalamotomy for tremor in the magnetic resonance imaging era. J Neurosurg 2013;118:713-8.

23 Niranjan A, Raju SS, Kooshkabadi A, et al. Stereotactic radiosurgery for essential tremor: retrospective analysis of a 19-year experience. Mov Disord 2017:32:769-77.

24 Tuleasca C, Witjas T, Najdenovska E, et al. Assessing the clinical outcome of VIM radiosurgery with voxel-based morphometry: visual areas are linked with tremor arrest! Acta Neurochir 2017;159:2139-44.

25 Halpern CH, Santini V, Lipsman N, et al. Three-Year follow-up of prospective trial of focused ultrasound thalamotomy for essential tremor. Neurology 2019;93:e2284-93.

26 Leksell Gamma Knife Society. Leksell gamma knife treatment statistics report 19682018. Stockholm: Leksell Gamma Knife Society, 2019.

27 FUS Foundation. Patient stories [Internet]. Available: https://www.fusfoundation.org/ for-patients/patient-stories [Accessed cited 2020 May 15].

28 Lipsman N, Schwartz ML, Huang Y, et al. MR-guided focused ultrasound thalamotomy for essential tremor: a proof-of-concept study. Lancet Neurol 2013;12:462-8.

29 Elias WJ, Huss D, Voss T, et al. A pilot study of focused ultrasound thalamotomy for essential tremor. N Engl J Med 2013:369:640-8.

30 Chang WS, Jung HH, Kweon EJ, et al. Unilateral magnetic resonance guided focused ultrasound thalamotomy for essential tremor: practices and clinicoradiological outcomes. J Neurol Neurosurg Psychiatry 2015;86:257-64.

31 Gallay MN, Moser D, Rossi F, et al. Incisionless transcranial MR-guided focused ultrasound in essential tremor: cerebellothalamic tractotomy. J Ther Ultrasound 2016:4:5-10.

32 Kim M, Jung NY, Park CK, et al. Comparative evaluation of magnetic resonanceguided focused ultrasound surgery for essential tremor. Stereotact Funct Neurosurg 2017:95:279-86.

33 Zaaroor M, Sinai A, Goldsher D, et al. Magnetic resonance-guided focused ultrasound thalamotomy for tremor: a report of 30 Parkinson's disease and essential tremor cases. J Neurosurg 2018;128:202-10. 